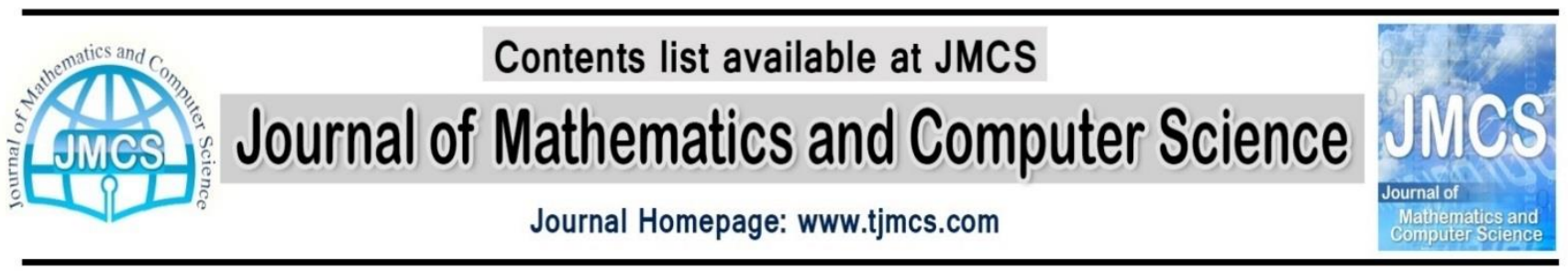

\title{
A Fictitious Time Integration Method for a One-Dimensional Hyperbolic Boundary Value Problem
}

\author{
Mir Sajjad Hashemi ${ }^{1, *}$, Maryam Sariri ${ }^{1}$ \\ ${ }^{1}$ Department of Mathematics, Basic Science Faculty, University of Bonab, Bonab 55517, Iran \\ *hashemi_math396@yahoo.com,hashemi@bonabu.ac.ir
}

\begin{abstract}
Article history:
Received July 2014

Accepted August 2014

Available online November 2014
\end{abstract}

\begin{abstract}
This paper gives an estimate for the initial-boundary value problem of wave equations by using the Fictitious Time Integration Method (FTIM) previously developed by Liu and Atluri [1]. Given examples confirm that FTIM is highly efficient approach to find the true solutions. It is interesting that the FTIM can easily treat the boundary value problems without any iteration and has high efficiency and high accuracy.
\end{abstract}

Keywords: Wave Equation, method of line, Fictitious Time Integration Method, Group preserving scheme, Lie group, Geometric numerical integration.

\section{Introduction}

Partial differential equations (PDEs) are first divided into two categories: non-evolutionary and evolutionary. Then, the latter is further classified into as parabolic and hyperbolic types according to the number of real characteristic lines. A non-evolutionary PDE is usually named elliptic type PDE because it exists no real characteristic line. Our task is to develop a non-iterative algorithm having the advantages of easy to numerical implementation, and a great flexibility applying to the most parabolic type boundary value problems (BVPs) without resorting on special treatments. This paper is motivated by using the evolutionary property of parabolic type PDE and the accurcay of numerical time integrators, and proposes a natural and mathematical equivalent approach to transform the underlying equation into a parabolic PDE without destroying the original structure.

In this paper, firstly by transforming the dependent variable $u(x, t)$ into a new one by $v(x, y, t):=(1+t) u(x, t)$ such that the original equation is naturally and mathematically equivalently written as a quasilinear parabolic equation, we use the method of line for semi-discretization of wave 
equation and then we apply the GPS, which firstly derived by Liu [2]. GPS uses the Cayley transformation and the Pade' approximations in the augmented space, namely Minkowski. After that, Liu [3] applied the GPS for backward heat conduction problems, which is an ill-posed problem and considered its stability and showed that obtained results is better than the Galerkin solutions. Lee et al. [4] for solving the initial value problems of stiff ordinary differential equations have proposed a modified GPS. Abbasbandy and Hashemi have developed a combination of method of line and GPS to obtain the solution of a severely ill-posed Laplace equation [5]. Also a combination of GPS and Lie symmetries are introduced by Hashemi et.al in [6]. The dynamic behavior of a single-mass, two degree of freedom bilinear oscillator has considered by Liu [7], by using the GPS. Estimation of the temperature-dependent thermal conductivity of a one-dimensional inverse heat conduction problem has studied by one-step GPS in $[8]$.

One of the most imortant hyperbolic second-order equations is the wave equation

$$
u_{t t}-c^{2} u_{x x}=0
$$

where $x$ signifies the spatial variable, $t$ the time variable, $u=u(x, t)$ the unknown function and $c$ is a given positive constant. The wave equation describes vibrations of a string. Physically $u(x, t)$ represents the value of the normal displacement of a particle at position $x$ and time $t$.

Our task is to develop a non-iterative algorithm having the advantages of easy to numerical implementation, and a great flexibility applying to the most hyperbolic type BVPs without resorting on special treatments. Let us begin with a discussion of the following quasilinear hyperbolic equation:

$$
\begin{gathered}
\Delta u(x, t)=F\left(x, t, u, u_{x}, u_{t}, \cdots\right), \quad(x, t) \in \Omega, \\
u(x, t)=H(x, t), \quad(x, t) \in \Gamma,
\end{gathered}
$$

where $\Delta:=\frac{\partial^{2}}{\partial t^{2}}-c^{2} \frac{\partial^{2}}{\partial x^{2}}, \Gamma$ is the boundary of the problem domain $\Omega$, and $F$ and $H$ are given functions. It is known that for the evolutionary type PDEs a semi-discretization of the spatial coordinates together with numerical time integrators for initial value problems (IVPs) can help us to find numerical solutions effectively.

\section{A fictitious time integration approach}

First we propose the following transformation:

$$
v(x, t, \tau)=(1+\tau) u(x, t),
$$

and introduce a viscosity damping coefficient $v$ in Eq. (1):

$$
0=v \Delta u-v F\left(x, t, u, u_{x}, u_{t}, \ldots\right)
$$

Multiplying the above equation by $1+\tau$ and using Eq. (5) we have 


$$
0=v \Delta v-v(1+\tau) F\left(x, t, u, u_{x}, u_{t}, \ldots\right)
$$

Recalling that $\frac{\partial v}{\partial \tau}=u(x, t)$ by Eq. (5), and adding it on both the sides of the above equation we obtain

$$
\frac{\partial v}{\partial \tau}=v \Delta v-v(1+\tau) F\left(x, t, u, u_{x}, u_{t}, \ldots\right)+u
$$

Finally by using $u=v /(1+t), u_{x}=v_{x} /(1+t)$ and $u_{t}=v_{t} /(1+t)$, etc. we can change Eqs. (1) and (2) into a parabolic type PDE:

$$
\begin{gathered}
\frac{\partial v}{\partial \tau}=v \Delta v-v(1+\tau) F\left(x, t, \frac{v}{1+\tau}, \frac{v_{x}}{1+\tau}, \frac{v_{t}}{1+\tau}, \ldots\right) \quad(x, t) \in \Omega, \\
v(x, t, \tau)=(1+\tau) H(x, t), \quad(x, t) \in \Gamma .
\end{gathered}
$$

There is maybe another selection of Eq. (5) by using $v(x, t, \tau)=q(\tau) u(x, t)$, where we require that $\mathrm{q}(0)=1$. However, when $q(\tau)$ is more complex than $1+\tau$ the resulting PDE is more complex than Eq. (9), and there seems no good reason to select a more complex $q(\tau)$. The above idea has been proposed and used by Liu and his coworkers in [9-15], whose numerical results are very well and satisfactory.

\subsection{Semi-discretization}

Let $v_{i, j}(\tau):=v\left(x_{i}, t_{j}, \tau\right)$ be a numerical value of $v$ at the grid point $\left(x_{i}, t_{j}\right) \in \Omega$, and at the fictitious variable $\tau$. Applying a semi-discrete procedure on the Eq. (8), yields a coupled system of ordinary differential equations (ODEs):

$$
\begin{aligned}
& v_{i, j}^{\prime}=\frac{v}{(\Delta x)^{2}}\left[v_{i+1, j}-2 v_{i, j}+v_{i-1, j}\right]-\frac{v}{(\Delta t)^{2}}\left[v_{i, j+1}-2 v_{i, j}+v_{i, j-1}\right] \\
& +\frac{v_{i, j}}{1+\tau}-v(1+\tau) F\left(x_{i}, t_{j}, \frac{v_{i, j}}{1+\tau}, \frac{v_{i+1, j}-v_{i-1, j}}{2(1+\tau) \Delta x}, \frac{v_{i, j+1}-v_{i, j-1}}{2(1+\tau) \Delta t}, \cdots\right),
\end{aligned}
$$

where $\Delta x$ and $\Delta t$ are uniform spatial grid lengths in $x$ and $t$ directions, and $m$ is the number of subintervals in each direction, assuming the same.

In this section we have transformed the boundary value problem of the one-order hyperbolic PDE in Eq. (1) to an evolutionary problem of a parabolic PDE in Eq. (8), and finally arrived to an initial value problem in the n-dimensional ODE system (10) with dimensions $n=m^{2}$. The initial value of Eq. (10) is given through a guess because the true initial condition of $v(x, t, 0)=u(x, t)$ is not known a priori; however, when $\left(x_{i}, t_{j}\right)$ is located on the boundary, the boundary condition (9) has to be satisfied. 


\section{GPS for differential equations system}

Upon letting $v=\left(v_{1,1}, v_{1,2}, \cdots, v_{m, m}\right)^{T}$ and $f$ denoting a vector with the $i j$-th component being the right-hand side of Eq. (10) we can write it as a vector form:

$$
v^{\prime}=f(v, \tau), \quad v \in R^{n}, \quad \tau \in R
$$

where $n$ is the number of total grid points inside the domain $\Omega$. GPS can preserve the internal symmetry group of the considered ODE system. Although we do not know previously the symmetry group of differential equations system, Liu [2] has embedded it into an augmented differential system, which concerns with not only the evolution of state variables themselves but also the evolution of the magnitude of the state variables vector. We note that

$$
\|v\|=\sqrt{v^{T} v}=\sqrt{v \cdot v}
$$

where the superscript $\mathrm{T}$ signifies the transpose, and the dot between two $\mathrm{n}$-dimensional vectors denotes their inner product. Taking the derivatives of both the sides of Eq. (12) with respect to t, we have

$$
\frac{d\|v\|}{d t}=\frac{(\dot{v})^{T} v}{\sqrt{v^{T} v}} .
$$

Then, by using Eqs. (11) and (13) we can derive

$$
\frac{d\|v\|}{d t}=\frac{f^{T} v}{\|v\|} .
$$

It is interesting that Eqs. (11) and (14) can be combined together into a simple matrix equation:

$$
\frac{d}{d \tau}\left[\begin{array}{c}
v \\
\|v\|
\end{array}\right]=\left[\begin{array}{cc}
0_{n \times n} & \frac{f(v, \tau)}{\|v\|} \\
\frac{f^{T}(v, \tau)}{\|v\|} & 0
\end{array}\right]\left[\begin{array}{c}
v \\
\|v\|
\end{array}\right] .
$$

It is obvious that the first row in Eq. (15) is the same as the original equation (11), but the inclusion of the second row in Eq. (15) gives us a Minkowskian structure of the augmented state variables of $X:=\left(v^{T},\|v\|\right)^{T}$, which satisfies the cone condition:

$$
X^{T} g X=0
$$

where

$$
g=\left(\begin{array}{cc}
I_{n} & 0_{n \times 1} \\
0_{1 \times n} & -1
\end{array}\right) .
$$


is a Minkowski metric, and $I_{n}$ is the identity matrix of order $n$. In terms of $(v,\|v\|)$, Eq. (16) becomes

$$
X^{T} g X=v \cdot v-\|v\|^{2}=\|v\|^{2}-\|v\|^{2}=0 .
$$

It follows from the definition given in Eq. (12), and thus Eq. (16) is a natural result. Consequently, we have an $n+1$ dimensional augmented system:

$$
X^{\prime}=A X
$$

with a constraint (17), where

$$
A:=\left(\begin{array}{cc}
0_{n \times n} & \frac{f(v, \tau)}{\|v\|} \\
\frac{f^{t}(v, \tau)}{\|v\|} & 0
\end{array}\right)
$$

satisfying

$$
A^{T} g+g A=0
$$

is a Lie algebra $\operatorname{so}(n, 1)$ of the proper orthochronous Lorentz group $\operatorname{SOo}(n, 1)$. This fact prompts us to devise the group-preserving scheme (GPS), whose discretized mapping $G$ must exactly preserve the following properties:

i: $G^{T} g G=g$,

ii: $\operatorname{det} G=1$,

iii: $G_{0}^{0}>0$,

where $G_{0}^{0}$ is the 00 -th component of $G$. Although the dimension of the new system is raised one more, it has been shown that the new system permits a GPS given as follows:

$$
X_{l+1}=G(l) X_{l}
$$

where $X_{l}$ denotes the numerical value of $X$ at $\tau_{l}$, and $G(l) \in S O_{0}(n, 1)$ is the group value of $G$ at $\tau_{l}$ . The Lie group can be generated from $A \in \operatorname{so}(n, 1)$ by an exponential mapping,

$$
\exp [\Delta \tau A(l)]=\left[\begin{array}{cc}
I_{n}+\frac{\left(\alpha_{l}-1\right)}{\left\|f_{l}\right\|^{2}} f_{l} f_{l}^{T} & \frac{\beta_{l} f_{l}}{\left\|f_{l}\right\|} \\
\frac{\beta_{l} f_{l}^{T}}{\left\|f_{l}\right\|} & \alpha_{l}
\end{array}\right],
$$


where

$$
\begin{aligned}
& a_{l}:=\cosh \left(\frac{\Delta \tau\left\|f_{l}\right\|}{\left\|v_{l}\right\|}\right), \\
& b_{l}:=\sinh \left(\frac{\Delta \tau\left\|f_{l}\right\|}{\left\|v_{l}\right\|}\right) .
\end{aligned}
$$

Substituting Eq. (23) for $G(l)$ into Eq. (22), we obtain

$$
v_{l+1}=v_{l}+\eta_{l} f_{l}
$$

where

$$
\eta_{l}=\frac{\left(\alpha_{l}-1\right) f_{l} \cdot v_{l}+\beta_{l}\left\|v_{l}\right\|\left\|f_{l}\right\|}{\left\|f_{l}\right\|^{2}} .
$$

\section{Numerical procedure}

Starting from an initial value of $v_{i, j}$ which can be guessed in a rather free way, we employ the above GPS to integrate Eq. (11) from $\tau=0$ to a selected final time $\tau_{f}$. In the numerical integration process we can check the convergence of $v_{i, j}$ at the $l$ and $l+1$-steps by

$$
\sqrt{\sum_{i, j=1}^{m}\left[v_{i, j}^{l+1}-v_{i, j}^{l}\right]^{2}} \leq \varepsilon,
$$

where $\varepsilon$ is a selected convergent criterion. If at a time $\tau_{0} \leq \tau_{f}$ the above criterion is satisfied, then the solution of $u$ is given by

$$
u_{i, j}=\frac{v_{i, j}\left(\tau_{0}\right)}{1+\tau_{0}} .
$$

In practice, if a suitable $\tau_{f}$ is selected we find that the numerical solution is also approached very well to the true solution, even the above convergent criterion is not satisfied. The viscosity coefficient introduced in Eq. (5) can increase the stability of numerical integration. In particular we would emphasize that the present method is very stable and effectively without needing of any iteration. Below we give numerical examples to display some advantages of the present FTIM.

\section{Numerical examples}

In this section, we consider two examples of wave equation to exhibit the efficiency and power of FTIM. 
Example 1. We first consider a wave equation as follows:

$$
\begin{cases}u_{t t}=u_{x x}, & \\ u(x, 0)=x, & u_{t}(x, 0)=\cos (x) \\ u_{x}(0, t)=1, & u_{x}(\pi, t)=1\end{cases}
$$

The domain is given by

$$
\Omega=\{(x, t) \mid 0 \leq x \leq \pi, 0 \leq t \leq \pi\},
$$

and analytical solution is:

$$
u(x, t)=x+\cos x \sin t
$$

The boundary data of $H(x, t)$ in FTIM can be written as

$$
\begin{gathered}
v_{0, j}(\tau)=(1+\tau) \sin t_{j}, \quad v_{m+1, j}(\tau)=(1+\tau)\left(\pi-\sin t_{j}\right) \\
v_{i, 0}(t)=(1+\tau) x_{i}, \quad v_{i, m+1}(\tau)=(1+\tau) x_{i}
\end{gathered}
$$

We fix $\Delta x=\Delta t=\frac{1}{m}$ with $m=50$, which the number of equations in Eq. (10) will be $n=49 \times 49$. We start by an initial value of $v_{i, j}=1$ and integrate Eq. (30) by using the GPS with a time stepsize $\Delta \tau=0.001$ The final time is $\tau_{f}=10$. The values $v=0.1$ and $\varepsilon=0.0001$ are selected in this equation. Fig. 1. shows that obtained approximate solution is an accurate solution.
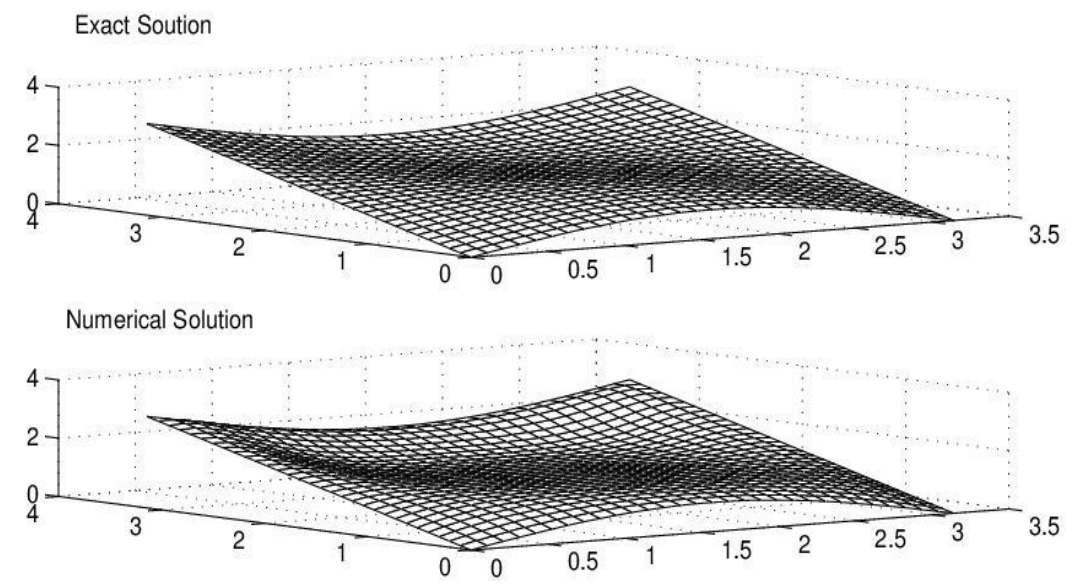

Figure 1: Plotting the numerical and Exact solution of Example 1 for a Wave equation. 
Example 2. Consider

$$
\left\{\begin{array}{l}
u_{t t}=u_{x x}, \\
u(x, 0)=0, \quad u_{t}(x, 0)=\sin (x), \\
u(0, t)=1, \quad u(\pi, t)=1,
\end{array}\right.
$$

with analytical solution

$$
u(x, t)=\sin x \sin t
$$

In the given domain

$$
\Omega=\{(x, t) \mid 0 \leq x \leq \pi, 0 \leq y \leq \pi\} .
$$

The boundary data $H(x, t)$ can be written as:

$$
\begin{gathered}
v_{0, j}(\tau)=0, \quad v_{m+1, j}(\tau)=0, \\
v_{i, 0}(t)=0, \quad v_{i, m+1}(\tau)=0 .
\end{gathered}
$$

We fix $\Delta x=\Delta t=\frac{1}{m}$ with $m=50$, and the number of equations in Eq. (10) is $n=49 \times 49$. We start by an initial value of $v_{i, j}=1$ for the interior nodes and integrate Eq. (32) by using the GPS with a time stepsize $\Delta \tau=0.0001$ and final time $\tau_{f}=10$. Under a given $v=0.3$ and $\varepsilon=0.0001$ the convergence is performed within the range of $\tau<\tau_{f}=10$. Finally, accuracy and powern of FTIM can be seen from the Fig. 2. 

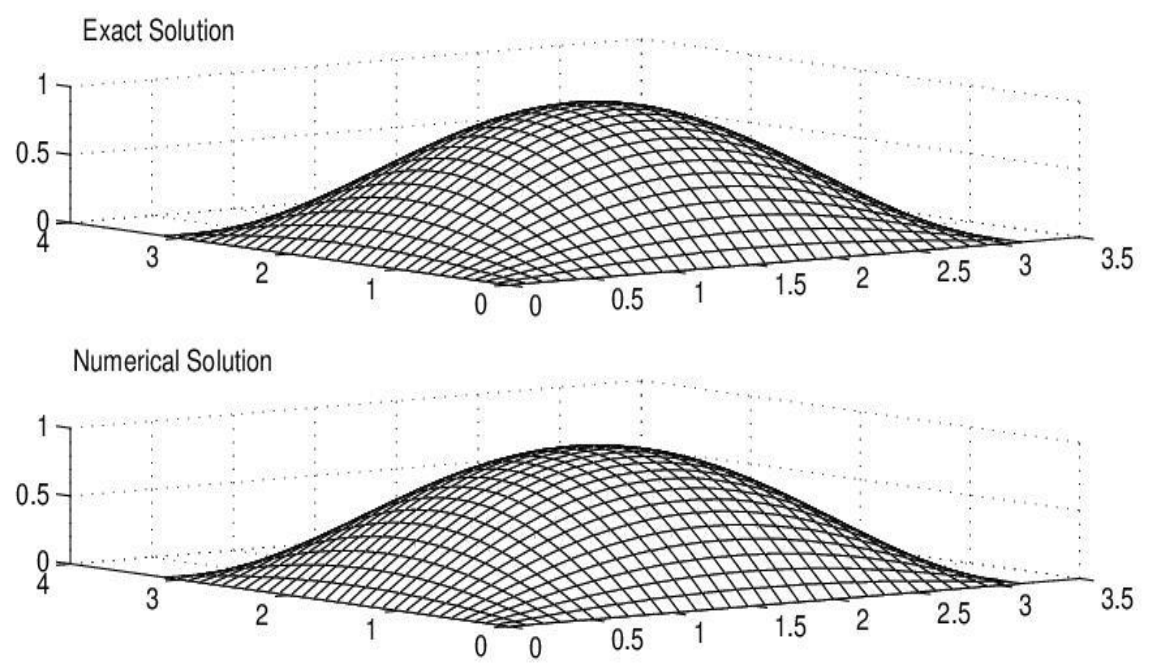

Figure 2: Plotting the numerical and Exact solution of Example 2 for a Wave equation.

\section{Conclusion}

In the present paper, the original quasilinear elliptic equation is mathematically transformed into a parabolic type evolutionary equation by in troducing a fictitious time coordinate, and adding a viscous damping coefficient to enhance the stability of numerical integration of the discretized equations by using a group preserving scheme. We must stress that the resulting parabolic equation is mathematically equivalent to the original equation, and no approximation is made. Hence, the present FTIM can work very effectively and accurately for the solution of boundary value problem of quasilinear elliptic equation. Because no iteration is required, the present method is very time saving and power of method is shown graphically.

\section{References}

[1] C.-S. Liu, S.N. Atluri, “A novel time integration method for solving a large system of non-linear algebraic equations", CMES 31 (2008) 71-83.

[2] C.-S. Liu, "Cone of non-linear dynamical system and group preserving schemes", Int. J. NonLinear Mech. 36 (2001) 1047-1068.

[3] C.-S. Liu, "Group preserving scheme for backward heat conduction problems", Int. J. Heat Mass Transfer 47 (2004) 2567-2576.

[4] H.-C. Lee, C.-K. Chen, C.-I. Hung, "A modified group-preserving scheme for solving the initial value problems of stiff ordinary differential equations", Appl. Math. Comput. 133 (2002) 445-59.

[5] S. Abbasbandy, M.S. Hashemi, "Group preserving scheme for the Cauchy problem of the Laplace equation", Eng. Anal. Bound. Elem. 35 (2011) 1003-1009.

[6] M.S. Hashemi, M.C. Nucci, S. Abbasbandy, "Group analysis of the modified generalized Vakhnenko equation", Commun. Nonlinear Sci. Numer. Simulat. 18 (2013) 867-877. 
[7] C.-S. Liu, "Two-dimensional bilinear oscillator: group-preserving scheme and steady-state motion under harmonic loading", Int. J. Non-Linear Mech. 38 (2003) 1581-1602.

[8] C.-S. Liu, "One-step GPS for the estimation of temperature-dependent thermal conductivity", Int. J. Heat Mass Transfer 49 (2006) 3084-3093.

[9] C.-S. Liu, "A fictitious time integration method for two-dimensional quasilinear elliptic boundary value problems", CMES: Computer Modeling in Engineering \& Sciences, 33 (2008) 179-198.

[10] C.-S. Liu, "A fictitious time integration method for solving the discretized inverse SturmLiouville problems, for specified eigenvalues", CMES: Computer Modeling in Engineering \& Sciences, 36 (2008) 261-286.

[11] C.-S. Liu, "A fictitious time integration method for solving m-point boundary value problems", CMES: Computer Modeling in Engineering \& Sciences, 39 (2009) 125-154.

[12] C.-S. Liu, "A fictitious time integration method for a quasilinear elliptic boundary value problem defined in an arbitrary plane domain", CMC: Computers, Materials \& Continua, 11 (2009) 15-32.

[13] C.-S. Liu, S. N. Atluri, "A novel time integration method for solving a large system of nonlinear algebraic equations", CMES: Computer Modeling in Engineering \& Sciences, 31 (2008) 71-83.

[14] C.-S. Liu, S. N. Atluri, "A fictitious time integration method (FTIM) for solving mixed complementarity problems with applications to non-linear optimization", CMES: Computer Modeling in Engineering \& Sciences, 34 (2008) 155-178.

[15] C.-S. Liu, S. N. Atluri, "A fictitious time integration method for the numerical solution of the Fredholm integral equation and for numerical differentiation of noisy data, and its relation to the filter theory", CMES: Computer Modeling in Engineering \& Sciences, 41 (2009) 243-261. 